\title{
Non-linear laminar flow into eccentrically placed well
}

\author{
K. K. UPADHYaY (*) \\ Received on September 2nd, 1975
}

\begin{abstract}
SUMmarr. - In steady state condition, non-linear laminar flow of fluid into an eccentrically placed well is considered. Its influence on the discharge and the dependence on related pliysical quantities is investigated. It is observed that as the well approaches towards the contour of intake, the discharge increases, which is an obvious result consistent with that obtained by I'olubarinova-Kochina in case of laminar flow. As a particular case, result for concentric well has also been deduced.
\end{abstract}

Rissunto. - Viene preso in considerazione un flusso di fluido laninare non-lineare - in condizioni di stato stazionario - dentro un pozzo disposto eccentricamente. Si è inoltre studiata sia la sua influenza sul getto che la dipendenza dalle relative quantità fisiche. $\dot{H}$ stato osservato che, come ci si avvicina al contorno dello sbocco, il flusso aumenta, il clıe è un risultato ovvio, in accordo con quanto ottenuto dai ricercatori Polubarinova e Kochina, nel caso di un flusso laminare. Il risultato relativo ad un pozzo coneentrico non è quindi che un caso particolare del problema affrontato in questa nota.

\section{1. - INTRODUCTION}

The intricacy in the nature of porous merlia does not always justify the natural flow of fluid through it to be purely laminar. However, it appear's more justifiable to consirler the flow through porous merlia to be either non-linear laminar or turbulent $\left(^{1}\right)$. Consequently Jain and Uparlhyay ( $\left.{ }^{2}\right)$, Elenbaas and Katz $\left({ }^{3}\right)$, Engelund $\left({ }^{4}\right)$ obtained specific solutions of some non-linear laminar and turbulent flow problems.

In the present paper, we consider the non-linear laminar stealy state flow of fluid into an eccentrically places well fully penetrating

(*) 79, Mill Road, Dewas, (M.P.) India 
the porous aquifer. It is found that the flow pattern is characterised by two different zones, in which discharge exhibits opposite character as regards its dependence on grain size of the medium, viscosity of the fluid and radius of the well. Further, it is observed that as the well approaches the contour of intake, the discharge increases abruptly as compared to that into a concentrically placed well, which is obvious from physical considerations.

The results for a concentric well have been deduced and compared with those obtained by Upadhyay $\left({ }^{5}\right)$.

\section{2. - Equations of Flutd flow in porous medium}

The Darey's law governing the laminar flow of fluid in porous media is

$$
v=k \frac{\mathrm{d} h}{\mathrm{~d} s}
$$

where $v, l$ and $\frac{d h}{d_{0}}$ denote the seepage velocity, seepage coefficient and hydraulic gradient respectively; flow being in the opposite direction of increasing $h$.

In case of an ecentrically placed circular well fully penetrating the cylindrical stratum (radius $R$ ) of unit thickness, the pressure $p$ at any point with complex coordinate $z$ is obtained in the form $\left(^{6}\right)$

$$
p=\frac{Q \mu}{2 \pi l_{i 0}} \log \frac{R\left(z-z_{1}\right)}{\left(K^{2}-z \bar{z}_{1}\right)}+C
$$

where $Q$, $k_{0}$ and $\mu$ represent flow rate, permeability of the medium and viscosity of the fluid respectively; $z_{1}$ denotes the centre of well and $\bar{z}_{1}$ is the corresponding inverse point. The constant $C$ is to be determined by boundary conditions.

Besides relations [1] and [2], the law for non-linear laminar flow is $\left(^{6}\right)$

$$
\frac{\mathrm{d} h}{\mathrm{~d} s}=a v+b v^{2}
$$

where $a$ and $b$ are constants. According to Engelund $\left(^{4}\right)$ 


$$
\begin{aligned}
& a=\frac{2000 u}{\varrho g d^{2}} \\
& b=\frac{35}{g d}
\end{aligned}
$$

$\varrho$ and $d$ being density of the fluid and grain size of the medium.

\section{3. - Formulation of the PROBLEM}

In steady state condition, we consider the flow of fluid into an uncased circular cylindrical well of radius $r_{w}$ eccentrically established at a distance $R_{1}$ from the centre of the contour of intake. It is assumed that the well is completely penetrating the porous aquifer of thickness $T$. The aquifer is considered to be homogeneous and isotropic bounded by horizontal impervious layers. The pressure at the contour of well and at the contour of intake are prescribed as $p_{w}$ and $p_{c}$ respectively. Let $r$ be the radial distance measured from the axis of intake.

As the effect of non-linear laminar or turbulent flow is observed to be appreciable event if such flow is restricted to a comparatively narrow zone $\left({ }^{4}\right)$, we consider the flow to be non-linear laminar within a narrow cylindrical zone of radius $r_{t}$ surrounding the well and laminar beyond this zone. Let the pressure at the transition boundary is $p_{\iota}$ [Fig. 1].

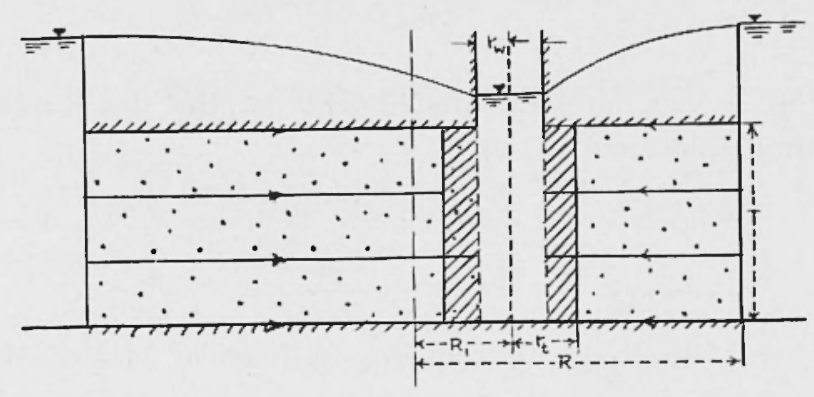

Fig. 1

In the present situation, we have

$$
\left(z_{1}\right)=\left(z_{1}\right)=R_{1}
$$


Therefore, expression for pressure distribution in the laminar zone (cf. [2]) takes the form

$$
p=\frac{Q \mu}{2 \pi \bar{k}_{0} \bar{T}} \log \frac{R\left(r-R_{1}\right)}{R^{2}-r R_{1}}+C
$$

The problem is to examine the infuence of non-linear laminar flow on discharge of fluid and its dependence on the related physical quantities.

\section{4. - Solution}

As in the vicinity of well the lines of equal pressure are closed to circles, therefore, we assume the contour of well as one of the isobars close to the circle of radius $r_{w}\left(^{6}\right)$. Along the boundary of transition, which is close to the contour of well, pressure $p_{\imath}$ may be obtained from [5] by using the boundary conditions

$$
\begin{aligned}
& p=p_{c} \quad \text { at } \quad r=R, \\
& p=p_{c} \quad \text { at } \quad r=R_{1}+r_{t}, \quad\left(r_{t} \ll R_{1} \ll R\right) .
\end{aligned}
$$

Hence

$$
p_{t}=p_{c}+\frac{Q \mu}{2 \pi k_{0} T} \log \frac{R r_{t}}{R^{2}-R_{1}^{2}}
$$

Since $p=\varrho g h$, pressure distribution in the non-linear laminar zone is obtainable from [3] as

$$
\frac{1}{\varrho g} \frac{\mathrm{d} p}{\mathrm{~d} r}=a v+b v^{2}
$$

In general, discharge $Q$ from any cylindrical surface of radius $\lambda$ and height $T$ is

$$
Q=2 \pi \lambda T v
$$

Consequently, in this situation

$$
r=R_{1}+\lambda, \quad r_{w} \leqslant \lambda \leqslant r_{t},
$$


it follows from [8], [9] and [10] that

$$
\int_{p_{u}}^{p_{\iota}} \mathrm{d} p=\varrho g \int_{R_{1}+r_{\imath}}^{R_{1}+r_{\iota}}\left(\frac{a Q}{2 \pi \cdot T}+\frac{b Q^{2}}{4 \pi^{2} r^{2} T^{2}}\right) \mathrm{u} r
$$

i.e. $\left.p_{\iota}=p_{n}+\varrho g \mid \frac{a Q}{2 \pi T} \log \left(\frac{R_{1}+r_{t}}{R_{1}+r_{w}}\right)+\frac{b Q^{2}}{4 \pi^{2} T^{2}}\left(\frac{1}{R_{1}+r_{w}}-\frac{1}{R_{1}+r_{\imath}}\right)\right]$

At the boundary of transition from laminar to non-linear laminar flow, the relation between critical Reynold's number $\xi_{c}=0.07$ and critical velocity $v_{c}$ is given by $\left.{ }^{4}\right)$

$$
v_{c}=\frac{Q}{2 \pi r_{t} T}-\xi_{c} \cdot \frac{a}{b},
$$

where $\frac{d h}{d s}$ as given by [1] and [3] yield the same value. Accordingly

$$
\frac{v_{c}}{k}=a v_{c}\left(1+\frac{b}{a} v_{c}\right)
$$

or, $\frac{1}{k}=1.07 a$.

Since $k=\frac{k_{\mathrm{o}} g g}{\mu}$, it follows that

$$
\frac{\mu}{k_{\mathrm{o}}}=1.07 \mathrm{a} \varrho g
$$

Using [14] in [7] and then comparing with [12], we get

$$
\begin{array}{r}
\frac{p_{c}-p_{u}}{\varrho g}=\frac{a Q}{2 \pi T}\left\{\log \left(\frac{R_{1}+r_{t}}{R_{1}+r_{v}}\right)-1.07 \log \left(\frac{R r_{t}}{R^{2}-R_{1}^{2}}\right)\right\}+ \\
+\frac{b Q^{2}}{4 \pi^{2} T^{2}}\left(\frac{1}{R_{1}+r_{v}}-\frac{1}{R_{1}+r_{t}}\right)
\end{array}
$$

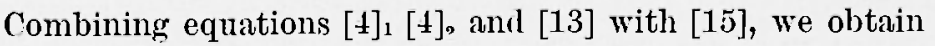

$$
\begin{gathered}
\frac{\varrho d^{3}\left(p_{c}-p_{w}\right)}{\mu^{2} r_{w}}=8000 \frac{r_{t}}{r_{w}}\left[\log \left(\frac{R_{1}+r_{t}}{R_{1}+r_{w}}\right)-1.07 \log \left(\frac{R r_{t}}{R^{2}-R_{1}^{2}}\right)+\right. \\
\left.+0.07 r_{t} \frac{\left(r_{t}-r_{w}\right)}{\left(R_{1}+r_{w}\right)\left(R_{1}+r_{t}\right)}\right]
\end{gathered}
$$


If we assume purely laminar flow in the entire flow regio $n$ then the flow rate $Q_{\text {lam }}$ may be obtained from [5] by using the corresponding boundary conditions at the well and the counter of intake. Hence

$$
Q_{l a m}-\frac{2 \pi k T}{\varrho g}\left[\frac{\left(p_{c}-p_{w}\right)}{\log \left(\frac{R^{2}-R_{\mathbf{1}}^{2}}{R r_{w}}\right)}\right]
$$

Therefore, from [13] and [17], we obtain the ratio

$$
\frac{Q}{Q_{\text {lam }}}=8560 \frac{r_{l}}{r_{w}}\left\{\frac{\mu^{2} r_{w}}{\varrho d^{3}\left(p_{c}-p_{w}\right)}\right\} \log \left(\frac{R^{2}-R_{1}^{2}}{R r_{w}}\right)
$$

Introducing dimensionless quantity $X$ and ratio $Y$ such that

$$
\begin{gathered}
X=\frac{\varrho d^{3}\left(p_{c}-p_{w}\right)}{\mu^{2} r_{w}}, \\
Y=\frac{Q}{Q_{\operatorname{lan}}}
\end{gathered}
$$

and combining [18] with [16], we obtain an implicit relation

$$
\begin{array}{r}
1.07 X=\frac{X Y}{Z}\left\lceil\log \left(\frac{R_{1}}{r_{w}}+\frac{X Y}{8560 Z}\right)-\log \left(\frac{R_{1}}{r_{w}}+1\right)+\right. \\
-1.07 \log \left(\frac{X Y}{8560 Z}\right)+ \\
\left.+1.07 Z+0.07 \frac{X Y}{8560 Z} \cdot \frac{\left(\frac{X Y}{8560 Z}-1\right)}{\left(\frac{R_{1}}{r_{w}}+1\right)\left(\frac{R_{1}}{r_{w}}+\frac{X Y}{8560 Z}\right)}\right],
\end{array}
$$

where $Z=\log \left(\frac{R^{2}-R_{1}^{2}}{R r_{w}}\right)$

It may be inferred from $[19]_{1}$ that the value of $X$ which is possible from physical considerations is $X>0$, hence equation [20] becomes 


$$
\begin{aligned}
& 1.07 Z=I\left[\log \left(\frac{R_{1}}{r_{w}}+\frac{X Y}{8560 Z}\right)-\log \left(\frac{R_{1}}{r_{v o}}+1\right)+\right. \\
& -1.07 \log \left(\frac{X Y}{8560 Z}\right)+ \\
& \left.+1.07 Z+\frac{0.07 X Y}{8560 Z} \cdot \frac{\left(\frac{X Y}{8560 Z}-1\right)}{\left(\frac{R_{1}}{r_{w}}+1\right)\left(\frac{R_{1}}{r_{w}}+\frac{X Y}{8560 Z}\right)}\right]
\end{aligned}
$$

\section{5. - Particular case}

If $R_{1}=0$ that is, when the well is established concentrically with respect to the contour of intake, equation [21] reduces to

$$
\begin{array}{r}
1.07 \log \left(\frac{R}{r_{w}}\right)=Y\left[0.07 \frac{X Y}{8560 \log \left(\frac{R}{r_{w}}\right)}+\right. \\
\left.-0.07 \log \left\{\frac{X Y}{8560 \log \left(\frac{R}{r_{w}}\right)}\right\}+1.07 \log \left(\frac{R}{r_{w}}\right)-0.07\right]
\end{array}
$$

which corresponds to the non-linear laminar flow of fluid into a fully penetrating concentric well discussed by Upadhyay $\left(^{5}\right)$.

\section{6. - Discussion}

From $[19]_{1}$, it is evident that $X$ depends on the density of the fluid, grain size of the medium, pressure difierence of the system, viscosity of the fluid and well radius. Since $d$ and $\mu$ occur in higher powers in expression for $X$, they highly affect the discharge. Moreover, from physical considerations it is obvious that $X$ and $Y$ are both positive. 
Now, to get the definite irlea of the result [21], we take $\frac{R}{r_{w}}=3 \cdot 10^{3}$ that is the radius of contour of intake is 3000 times the raclius of the well. Consiclering $\frac{R}{r_{w}}=10^{2}$, the numerical values of $Y$ are obtained corresponding to different values of $X>0$ and have been graphically plotterl in the form of curve - I [Fig. 2].

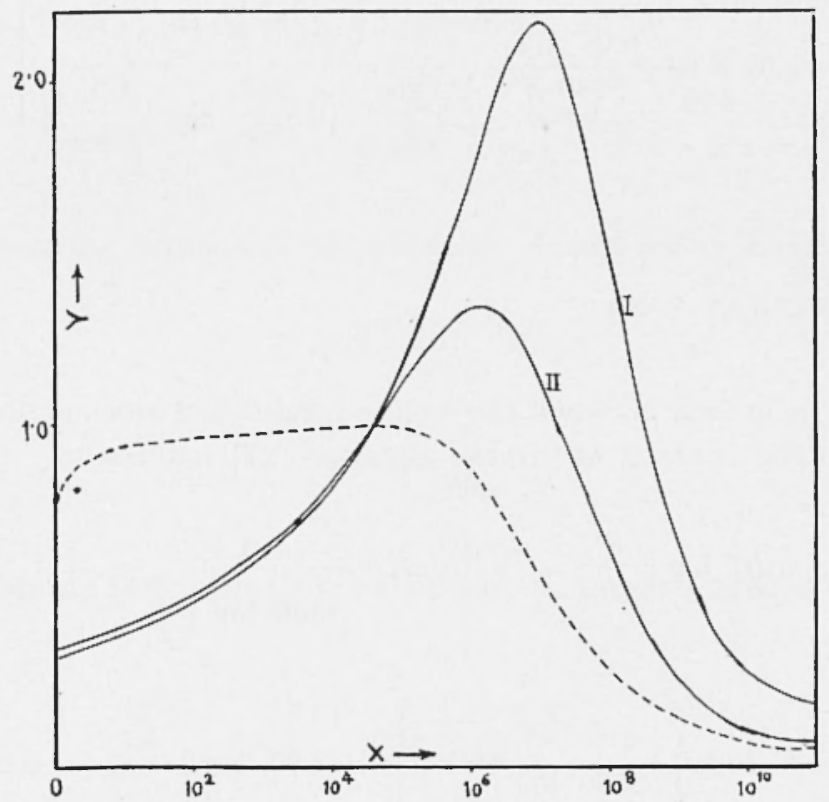

Fig. 2

It is seen from curva-I that as $X$ increases, initially $Y$ increases till it attains a maximum value 2.16 corresponcling to $X=1.2691 \cdot 10^{2}$, afterwards it descresases asymptotically. Thus in the former region $O<X<1.2691 \cdot 10^{7}$, the discharge increases as $X$ increases, that is, when the density of the fluid, grain size of the merlium and pressure difference of the system increases, viscosity of the fluid and the well rarlius decreases. In the later region $X>1.2691 \cdot 10^{2}$ the influence of non-linear laminar flow is reversed.

Thus, it may be concluded that in case of non-linear laminar flow, the flow pattern is characterised by two different zones in which, discharge exhibits opposite character. 


\section{7. - COMPARISION}

To examine as to how the position of the well affects the dischagre into it, we consider the cases $\frac{R_{1}}{r_{w}}=0$ and $\frac{R_{1}}{r_{w}}=10$. These cases have been graphically respresented by dotted curve and curve-II respectively in Fig. 2. Hence, it is inferred that as the well approaches the contour of intake the discharge increases abruptly as compared to that into a well concentrically established with respect to the contour of intake. From physical consideration, the result is quite obvious and consistent with that obtained by Poluberinova-Kochina $\left(^{6}\right)$ in case of laminar flow.

\section{ACKNowledGEMENT}

The author gratefully acknowledges the valuable guidance of Dr. S. K. Jain, Department of Applied Mathematics, G.S. Institute of Technology and Science, Indore in the preparation of this paper. He is also very much thankful to the referee for his valuable suggestions for the improvement of the paper.

\section{REFERENCES}

(1) Bear, J., 1972. - Dynamics of Auids in porous media. American Elsevier Publishing Company Inc., New York.

(2) JaIn, S. K., Upadiray, K. K., 1975. - Non-linear laminar flow of Auid into a partially penetrating well. "Gerlands Beiträge Zur Geophysik" (in press).

(3) Elenbaas, J. R., Katz, D. I., 1948. - A radial turbulent flow formula. "Trans. AIHE", 174, p. 25.

(4) Engelund, F., 1953. - On laminar and turbulent flou of ground water in homogeneous sand. "Trans. Dan. Acad. Tech. Sci", 3, pp. 1-105.

(5) VPadny ау, K. K., 1976. - Non-linear laminar flow of Auid into a fully penetrating cylindrical well. "Ind. J. Theo. Pliys." (in press).

(6) Polubarinova-Kochina, P. YA., 1952. - Theory of Ground water Movement. Princeton Lniversity, Press, Princeton, 364, p. 17. 